\title{
Sensor-based Characterization of Daily Walking: A New Paradigm in Pre-frailty/Frailty Assessment
}

Danya Pradeep Kumar ( $\nabla$ danyapradeep93@email.arizona.edu )

University of Arizona https://orcid.org/0000-0002-6913-5902

Nima Toosizadeh

University of Arizona

Jane Mohler

University of Arizona

Hossein Ehsani

University of Arizona

Cassidy Mannier

University of Arizona

Kaveh Laksari

University of Arizona

Research article

Keywords: Frailty, daily physical activity, wearable sensors, continuous walking, performance parameters.

Posted Date: April 7th, 2020

DOI: https://doi.org/10.21203/rs.2.16000/v3

License: (c) (i) This work is licensed under a Creative Commons Attribution 4.0 International License.

Read Full License 


\section{Abstract}

Background : Frailty is a highly recognized geriatric syndrome resulting in decline in reserve across multiple physiological systems. Impaired physical function is one of the major indicators of frailty. The goal of this study was to evaluate an algorithm that discriminates between frailty groups (non-frail and pre-frail/frail) based on gait performance parameters derived from unsupervised daily physical activity (DPA). Methods : DPA was acquired for 48 hours from older adults ( $\geq 65$ years) using a tri-axial accelerometer motion-sensor. Continuous bouts of walking for $20 \mathrm{~s}, 30 \mathrm{~s}, 40 \mathrm{~s}, 50 \mathrm{~s}$ and $60 \mathrm{~s}$ without pauses were identified from acceleration data. These were then used to extract qualitative measures (gait variability, gait asymmetry, and gait irregularity) and quantitative measures (total continuous walking duration and maximum number of continuous steps) to characterize gait performance. Association between frailty and gait performance parameters was assessed using multinomial logistic models with frailty as the dependent variable, and gait performance parameters along with demographic parameters as independent variables. Results : 126 older adults (44 non-frail, 60 pre-frail, and 22 frail, based on the Fried index) were recruited. Step- and stride-times, frequency domain gait variability, and continuous walking quantitative measures were significantly different between non-frail and pre-frail/frail groups ( $p$ $<0.05)$. Among the five different durations $(20 \mathrm{~s}, 30 \mathrm{~s}, 40 \mathrm{~s}, 50 \mathrm{~s}$ and $60 \mathrm{~s})$, gait performance parameters extracted from 60s continuous walks provided the best frailty assessment results. Using the $60 \mathrm{~s}$ gait performance parameters in the logistic model, pre-frail/frail group (vs. non-frail) was identified with $76.8 \%$ sensitivity and $80 \%$ specificity. Discussion : Everyday walking characteristics were found to be associated with frailty. Along with quantitative measures of physical activity, qualitative measures are critical elements representing the early stages of frailty. In-home gait assessment offers an opportunity to screen for and monitor frailty.

\section{Background}

Among the population who are 60 years or older, frailty is a highly recognized syndrome that is associated with decline in function and reserve across multiple physiologic systems (1-5). Frailty is characterized by a high vulnerability to adverse health outcomes such as disability, falls, hospitalization, institutionalization, and mortality (1). Reduction or impairment of physical function is a prime indicator of frailty (2), and frailty is one of the major reasons for falls in old age (6-12). Many definitions of frailty have been proposed: Fried et al used five criteria (slowness, exhaustion, weakness, low-activity and weight-loss) to identify frailty (2); Rockwood et al developed a frailty index based on impairments in cognitive status, mood, motivation, communication, mobility, balance, bowel and bladder function, activities of daily living, nutrition, social resources and number of comorbidities (13); Mitnitski et al constructed a frailty index based on 20 deficits as observed in a structural clinical examination based on the comprehensive geriatric assessment (CGA) (14); Jones et al also based their frailty index based on CGA which included 10 standard domains to construct a three level frailty index permitting risk stratification of future adverse outcomes (15); and Chin et al compared three different working definitions of frailty - inactivity plus low energy intake, inactivity plus weight-loss and inactivity plus low body mass 
index (16). Although many definitions of frailty have been proposed, we use Fried's frailty criteria as the most commonly implemented frailty assessment tool in our study. However, there is currently no objective method for assessing frailty that incorporates assessment of daily physical activity (DPA).

DPA data has been recently used to assess physical function, especially with the help of wearable sensor technology. Using wearable devices, it is possible to continuously measure DPA in the least intrusive manner and for longer durations of time in the participants' natural environment. Among several DPA, motion analysis of the trunk during walking is known to provide insights regarding neuromuscular deficits associated with frailty and aging (12). In our previous studies, among DPA measures (walking, standing, sitting, and lying), quantitative parameters related to walking, such as total walking duration and maximum number of steps, best discriminated between non-frail and pre-frail groups, with highest effect sizes for the number of steps and the percentage of walking duration within a 24-hour time period (17). While promising, we found that none of these outcomes (or their combination in a multinomial logistic analysis) could significantly discriminate between frailty groups when adjusted for age (17).

In this study, we aimed to improve detection of frailty-related neuromuscular deficits based on gait performance parameters derived from unsupervised DPA. Previous studies have used trunk motion data from supervised in-lab gait tests to characterize sensorimotor gait performance among frail elders including gait variability, asymmetry, initiation, and irregularity $(11,12,18-22)$. We hypothesized that using more robust measures of unsupervised DPA gait performance such as gait variability, asymmetry and irregularity (instead of number of steps) it would be possible to distinguish between non-frail and prefrail/frail older adults.

\section{Methods}

This observational cross-sectional study was performed at Arizona Center on Aging, Tucson, AZ. Participants in this study were from primary, secondary, and tertiary health-care settings within our academic network and also from community providers and aging service organizations. DPA was recorded from eligible volunteers for 48 hours and the walking data from the DPA was processed to study the gait performance parameters and associate these characteristics with frailty.

\section{Participants}

Older adults ( 65 years or older), without severe mobility disorder and the ability to walk at least $10 \mathrm{~m}$ with or without an assistive device, were considered eligible for the study. Participants with cognitiveimpairment (screened by a Mini-Mental State Examination (MMSE) (23) score of <23) or terminal illness were excluded. All the eligible participants signed a written consent form according to the principles expressed in the Declaration of Helsinki (24), approved by the Institutional Review Board of the University of Arizona. 


\section{Demographic and Clinical Measures}

The recorded clinical measures included self-reported history of falls, use of assistive device and the number of prescriptions. Interviewer-administered questionnaires included the MMSE, Mobility-Tiredness Scale (25), Center for Epidemiologic Studies Depression Scale (CES-D) (26), Falls Efficacy ScaleInternational (FES-I) (27), and Barthel Activity of Daily Living (ADL) Scale (28).

\section{Frailty Assessment}

Frailty was assessed using the five criteria proposed by Fried et al (2), including: self-reported weight loss, weakness measure by the grip strength, self-reported exhaustion, slowness measure by the walking test, and self-reported low energy expenditure. A score of one point was given for each criterion recorded, totaling a score in the range of 0-5. Frailty was categorized as follows: non-frail (score 0 ), pre-frail (score 1-2), and frail (score 3-5).

\section{Sensor-Based Daily Physical Activity Assessment}

DPA was quantified for two consecutive days (48 hours) using a tri-axial accelerometer sensor (PAMSys, BioSensics Cambridge, MA, USA) fixed in a tee-shirt, with a device pocket located at the sternum. PAMSys is a small $(5.1 \times 3 \times 1.6 \mathrm{~cm})$, light $(24 \mathrm{~g})$ recording system containing inertial sensors. PAMSys is an ambulatory system designed and developed for human motion analysis using a kinematic sensor. It uses one kinematic sensor placed on the chest and is capable of accurately identifying and recording postural transitions (sit-to-stand and stand-to-sit), laying, walking, and standing (29-31). PAMSys uses discrete wavelet transformation techniques based on a previously validated algorithm (PAMWare, BioSensics Cambridge, MA, USA) to identify walking bouts (32-34). Using this method, walking bouts were defined by a minimum of three successive steps (29), where steps were estimated by the detection of an acceleration peak beyond a predefined threshold after filtering the signal (31). Using this software several gait parameters were derived including: the duration of walking, walking bout times (duration of each walking episode), number of steps per walking bout, and walking cadence per bout. PAMWare is $87 \%$ sensitive and $87 \%$ specific for gait detection $(29,30)$. In the current study, we used raw vertical acceleration data recorded by the PAMSys tri-axial accelerometer sensor, as well as the timing of walking bouts from the PAMWare software to extract gait performance parameters within continuous walking bouts.

\section{Continuous Walking Bouts}

Previous studies that explored the gait characteristics of non-disabled adults for two weeks to define walking duration, found that $81 \%$ of all walking bouts lasted about 60 seconds $(35,36)$. Further, previous studies suggested that a duration of 60 -second continuous data would provide a reliable sample for nonlinear dynamic analysis (37). Accordingly, in our study, we used continuous walking bouts of 60 seconds or longer for the extraction of gait performance parameters. In addition to the 60s analysis, all the gait performance parameters were extracted for $20 \mathrm{~s}, 30 \mathrm{~s}, 40$ s and 50 s lengths of continuous walking 
bouts to investigate the effect of continuous walk length on data analysis and results. Gait performance parameters including time- and frequency-domain gait variability, gait asymmetry, and gait irregularity were extracted from these continuous walking bouts of all the durations, with no pauses longer than 1.7s between gait cycles (17). Allowable $1.7 \mathrm{~s}$ pause between gait cycles was conservatively selected based on the average plus standard deviation stride time duration observed in frail participants (17). All the sensorbased gait performance outcome measures are shown in Table 1.

For each continuous walking bout, the raw vertical acceleration signal extracted from the PAMSys sensor was filtered using a second order Butterworth filter (cut-off frequency of $2.5 \mathrm{~Hz}(38)$ ), and the peaks of the filtered acceleration signal were detected using a peak-detection algorithm. The time-interval between two consecutive peaks was defined as the step-time, and the time-interval between alternate peaks was defined as the stride-time.

Gait Variability: We defined gait variability as the stride-to-stride fluctuation in gait cycles, which has been associated with high risk of fall and cognitive impairments in elders (6,39-41). Gait variability reflects inconsistency in physiological systems that regulate walking, including neuromuscular, reflexive postural control, and cardiovascular systems (42). We used two methods to assess gait variability: 1) step- and stride-time variability using time-domain; and 2) power spectral density (PSD) using frequency-domain analysis $(43,44)$. Step- or stride-time variability was calculated as the coefficient of variation of the series of step- or stride-times for each continuous walking bout. For PSD analysis, the power spectrum of the acceleration data was calculated using Welch's averaged modified periodogram method (45), to represent the frequency components of the acceleration signal (46). We used a window size of 512 samples and an FFT length of 2-times the next higher power of the window size (45). An overlap of $50 \%$ was considered between the windows. The locomotion band between $0.5-3.0 \mathrm{~Hz}$ was analyzed (45). PSD components were extracted from the raw acceleration signal, including: maximum PSD peak, PSD width (full width at half maximum height), PSD slope (PSD width to the peak) and dominant walking frequency. A higher variability in walking was identified by a shorter and wider PSD peak.

Gait Asymmetry: When gait becomes less automatic due to sarcopenia and cognitive aging, left-right step coordination may require more effort, especially among frail individuals $(17,37,47)$. Further, studies showed that no strong association between gait variability and asymmetry exists, suggesting that asymmetry reflects an independent measure of gait impairments due to distinct pathological causes $(17,47)$. Here, step asymmetry was obtained from the autocorrelation function of the vertical acceleration signal $(17,47)$, represented by a sequence of autocorrelation coefficients over increasing time lags. (see Equation 1 in the Supplementary Files)

where and are the prominence of the first and the second peaks respectively after the central (zero lag) peak (48).

Gait Irregularity: Results from supervised gait studies showed that irregularity measures can describe predictability of walking cycles, which can be influenced by both neurological and neuromuscular diseases (49-52). Further, within in-lab settings, it has been demonstrated that gait irregularity can differ 
between non-frail and pre-frail older adults (21). We used Sample Entropy (SampEn) assessment defined as equation (2), where was defined as the number of matches in the filtered acceleration signal length (distance function smaller than tolerance ) and as the number of matches of length ( ) (53-57). (see Equation 2 in the Supplementary Files).

The time-delay of the signal was calculated using mutual information method for all the continuous walks (58), and the average time-delay of all the continuous walks was used to calculate the SamplEn for each volunteer. We used embedding dimension $\mathrm{m}=3$, and tolerance $r=0.2$ times the standard deviation of the signal, which are commonly used to compute sample entropy of gait signal (53-57).

Table 1. Sensor-based outcome measures

\begin{tabular}{|c|c|c|}
\hline Parameter & Description & Reference \\
\hline Step/stride time & Time-interval between two consecutive/alternate acceleration peaks & \\
\hline \multicolumn{3}{|l|}{ ait Variability } \\
\hline Step/stride time variability & $\begin{array}{l}\text { Coefficient of variation (\%), standard deviation of step/stride time } \\
\text { over mean step/stride time }\end{array}$ & $(17)$ \\
\hline PSD max & $\begin{array}{l}\text { Maximum height of the PSD distribution curve representing the } \\
\text { amount of walking that occurs at the dominant frequency }\end{array}$ & $(45,59)$ \\
\hline PSD width & $\begin{array}{l}\text { The width of the PSD curve at half of the maximum height } \\
\text { representing the range of walking frequencies }\end{array}$ & $(45,59)$ \\
\hline PSD slope & $\begin{array}{l}\text { The slope of the PSD curve from the peak to the width representing } \\
\text { the variability of walking. }\end{array}$ & $(45,59)$ \\
\hline Dominant frequency & $\begin{array}{l}\text { The frequency at which the PSD curve attains its peak, representing } \\
\text { the frequency at which most of the walking cycles occur }\end{array}$ & $(45,59)$ \\
\hline \multicolumn{3}{|l|}{ ait Asymmetry } \\
\hline & $\begin{array}{l}\text { Unbiased auto-correlation coefficients of gait signal, } \\
\text { representing left-right step coordination }\end{array}$ & $(11,60)$ \\
\hline \multicolumn{3}{|l|}{ ait Irregularity } \\
\hline \multicolumn{3}{|l|}{$\begin{array}{l}\text { ontinuous Walking Quantitative } \\
\text { easures }\end{array}$} \\
\hline No. of continuous walks & Total number of continuous walks in the 48 hour duration & \\
\hline $\begin{array}{l}\text { Total continuous walking } \\
\text { aration }\end{array}$ & Total duration of continuous walks in the 48 hour duration & \\
\hline Max walking bout & Maximum duration of continuous walking in 48 hours & \\
\hline Max no. of continuous steps & $\begin{array}{l}\text { Maximum number of continuous steps in the longest duration } \\
\text { continuous walking bout in } 48 \text { hours }\end{array}$ & \\
\hline Walking bout variability & $\begin{array}{l}\text { Coefficient of variation (\%), standard deviation of walking bouts over } \\
\text { mean walking bout }\end{array}$ & \\
\hline $\begin{array}{l}\text { Duration of non-continuous } \\
\text { alks (\% of total walking } \\
\text { aration) }\end{array}$ & $\begin{array}{l}\text { Duration of walks which were not continuous for } 60 \text { s or longer (total } \\
\text { duration of } 60 \text { s walking minus continuous walking with no pause) }\end{array}$ & \\
\hline
\end{tabular}

SD - Power Spectral Density

Continuous Walking Quantitative Measures: In addition to the above-mentioned features, we extracted the following parameters in each continuous walking event: maximum walking bout, maximum number of continuous steps, walking-bout variability (coefficient of variation in walking bouts duration within 48 hours), and total duration of continuous walks. Of note, the parameters extracted here were only obtained for continuous walking events with no pause of $1.7 \mathrm{~s}$ or longer, as described above. Duration of non- 
continuous walk (total duration of 60 s walking minus 60 s continuous walking with no pause) was obtained as a percentage of total duration of walking from the PAMsys sensor.

\section{Statistical Analysis}

Separate analysis of variance (ANOVA) models were performed to compare sociodemographic parameters between the three Fried frailty groups. To explore differences in gait performance parameters among frailty categories, univariate ANOVA models were used with each gait performance parameter as the dependent variable, and the Fried frailty categories (non-frail and pre-frail/ frail) as the independent variable. Subsequently, gait performance parameters were used in a single multivariable nominal logistic model to assess the association between frailty categories and DPA gait performance parameters. In this model we combined pre-frail and frail groups, due to the limited number of frail participants. The model was developed following these steps: 1) univariate nominal logistic model analysis of the gait performance parameters as independent variables was performed. Gait performance parameters with significant association with frailty were considered for subsequent steps; 2) collinearity between the various gait performance parameters was tested using the variance inflation factor (VIF) index. VIF value greater than 10 represented the presence of collinearity (63); and 3) gait performance and demographic parameters were selected based on Akaike information criterion (AIC) values. Participants who exhibited no 20/30/40/50/60 second continuous walks were automatically categorized as frail in the respective model. All analyses were done using JMP (Version 11; SAS Institute Inc., Cary, NC, USA), and statistical significance was concluded when $p<0.05$.

\section{Results}

\section{Demographic and Clinical Measures}

The study involved a total of 126 participants, among whom 44 were non-frail, 60 were pre-frail and 22 were frail according to the Fried frailty criteria (2). Table 2 shows demographic and clinical characteristics. Pre-frail/frail participants were significantly older than non-frail participants, used assistive devices more frequently, had higher BMI, perceived tiredness, and fear of falling score $(p<0.05)$ and lower performance scores in $\mathrm{ADL}(p<0.05)$.

Table 2. Demographic and clinical characteristics 


\begin{tabular}{|c|c|c|c|c|c|c|c|}
\hline \multirow{2}{*}{$\begin{array}{l}\text { Characteristics } \\
\text { Age (years) }\end{array}$} & \multicolumn{3}{|c|}{$\begin{array}{c}\text { Non-frail } \\
(\mathrm{N}) \\
(\mathrm{n}=44)\end{array}$} & \multicolumn{3}{|c|}{$\begin{array}{l}\text { Pre-frail/Frail }(\mathrm{P} / \mathrm{F}) \\
(\mathrm{n}=82)\end{array}$} & \multirow{2}{*}{$\frac{p \text {-value }}{\overline{\mathrm{N} \text { vs } \mathrm{P} / \mathrm{F}}}$} \\
\hline & 74.6 & \pm & 6.5 & 81.2 & \pm & 8.6 & \\
\hline Height (cm) & 161.7 & \pm & 6.9 & $161.3 \pm$ & & 9.5 & 0.797 \\
\hline Weight (kg) & 67.2 & \pm & 12.8 & 76.0 & \pm & 18.1 & 0.005 \\
\hline Body mass index & 25.7 & \pm & 4.5 & 29.2 & \pm & 6.5 & 0.002 \\
\hline Gender & & & & & & & 0.190 \\
\hline Male & & 6( & 3.6) & $19(23.2)$ & & & \\
\hline Female & & 38 & 6.4) & $63(76.8)$ & & & \\
\hline History of falls & & 13 & 9.5) & $37(45.1)$ & & & 0.085 \\
\hline Falls Efficacy Scale - International & 120.8 & \pm & 4.2 & $31.3 \pm$ & 11 & & $<0.001$ \\
\hline Use of assistive devices & & 4( & $.1)$ & $41 \quad(50.0)$ & & & $<0.001$ \\
\hline Mobility-tiredness scale & 5.6 & \pm & 0.8 & $4.1 \pm$ & 1. & & $<0.001$ \\
\hline MMSE & 29.2 & \pm & 1.1 & 28.6 & 1 . & & 0.060 \\
\hline CES-D & 6.6 & \pm & 5.7 & 8.9 & 7. & & 0.079 \\
\hline Barthel ADL Scale & & 7.6 & $\pm \quad 4.6$ & 93.8 & \pm & 7.9 & 0.004 \\
\hline
\end{tabular}

Results presented as mean \pm SD or number (\%). Bold-faced values show statistical significance $(p<0.05)$.

\section{Sensor-Based Daily Physical Activity Assessment}

Table 3 shows the sensor-based DPA parameters for 60 s continuous walking with resulting $p$-values of one-way ANOVA test (Figure 1, Table 3) and Cohen's d effect sizes. Supplementary Table 1 (a-d) shows the sensor-based DPA parameters for $20 \mathrm{~s}, 30 \mathrm{~s}, 40 \mathrm{~s}$, and 50 s continuous walks with resulting $p$-values of one-way ANOVA tests respectively.

Of note, among the total 126 participants, 4 non-frail (9.09\%), 17 pre-frail $(28.33 \%)$, and 11 frail (50\%) had no walking bout equal or longer than 60 seconds. These participants who did not have any $60 \mathrm{~s}$ continuous walking bout (age: $81.59 \pm 8.34)$ were significantly $(p=0.035)$ older than the participants who had continuous walks (age: 77.94 \pm 8.43 ). Frailty status was also significantly $(p=0.001)$ different between the participants who did not have any continuous walking bouts (87.5\% pre-frail/frail) compared to those who had continuous walks (57.45\% pre-frail/frail). However, the BMI and gender were not significantly different between these groups ( $p>0.079)$.

Table 3. Gait performance parameters for 60s continuous walks, one-way ANOVA results, and Cohen's $d$ effect sizes between non-frail(N) and pre-frail(P)/frail(F) groups 


\begin{tabular}{|c|c|c|c|}
\hline Parameter & $\begin{array}{c}\text { Non-frail } \\
(\mathrm{N}) \\
(\mathrm{n}=40) \\
\end{array}$ & $\begin{array}{c}\text { Pre-frail/Frail } \\
(\mathrm{P} / \mathrm{F}) \\
(\mathrm{n}=54) \\
\end{array}$ & $\frac{p \text {-value (Eff. size) }}{\text { N vs. } \mathrm{P} / \mathrm{F}}$ \\
\hline \multicolumn{4}{|l|}{$\overline{\text { Temporal Gait Parameters }}$} \\
\hline Step-time (s) & $0.56 \pm 0.05$ & $0.61 \pm 0.06$ & $<0.001(0.91)$ \\
\hline Stride-time (s) & $1.13 \pm 0.09$ & $1.23 \pm 0.12$ & $<0.001(0.92)$ \\
\hline \multicolumn{4}{|l|}{ Time Domain Gait Variability } \\
\hline Step variability (\%) & $10.79 \pm 2.80$ & $10.95 \pm 3.36$ & $0.812(0.05)$ \\
\hline Stride variability (\%) & $9.16 \pm 2.94$ & $8.74 \pm 3.04$ & $0.509(0.13)$ \\
\hline \multicolumn{4}{|l|}{ Frequency-domain Gait Variability } \\
\hline PSD max (W/Hz) & $0.17 \pm 0.16$ & $0.07 \pm 0.07$ & $<0.001(0.90)$ \\
\hline PSD width $(\mathrm{Hz})$ & $0.22 \pm 0.10$ & $0.21 \pm 0.03$ & $0.446(0.17)$ \\
\hline PSD slope $(\mathrm{W})$ & $1.24 \pm 1.21$ & $0.48 \pm 0.49$ & $<0.001(0.90)$ \\
\hline Dominant frequency $(\mathrm{Hz})$ & $1.90 \pm 0.16$ & $1.73 \pm 0.18$ & $<0.001(0.97)$ \\
\hline \multicolumn{4}{|l|}{ Gait Asymmetry } \\
\hline Asymmetry 1 & $1.10 \pm 0.14$ & $1.05 \pm 0.21$ & $0.229(0.26)$ \\
\hline Asymmetry 2 & $0.09 \pm 0.07$ & $0.08 \pm 0.06$ & $0.625(0.10)$ \\
\hline \multicolumn{4}{|l|}{ Gait Irregularity } \\
\hline Time delay (ms) & $145.39 \pm 14.90$ & $156.25 \pm 23.86$ & $0.124(0.33)$ \\
\hline Sample entropy (bits) & $0.93 \pm 0.28$ & $1.00 \pm 0.29$ & $0.225(0.25)$ \\
\hline \multicolumn{4}{|l|}{ Continuous Walk Quantitative Measures } \\
\hline Number of continuous walks & $13.25 \pm 11.22$ & $10.63 \pm 10.43$ & $0.112(0.33)$ \\
\hline Total continuous walking duration (s) & $4042.33 \pm 3012.86$ & $2436.79 \pm 1988.46$ & $0.001(0.70)$ \\
\hline Max walking bout (s) & $475.62 \pm 512.27$ & $216.98 \pm 228.95$ & $0.001(0.77)$ \\
\hline Max number of continuous steps & $1867.58 \pm 1735.98$ & $896.63 \pm 1055.53$ & $0.001(0.78)$ \\
\hline Walking bout variability (\%) & $252.74 \pm 110.51$ & $195.88 \pm 73.98$ & $0.002(0.69)$ \\
\hline Duration of non-continuous walks (\% total of walking duration) & $41.82 \pm 27.85$ & $47.25 \pm 42.39$ & $0.482(0.15)$ \\
\hline
\end{tabular}

PSD - Power Spectral Density

Among the 60s continuous gait parameters, frequency domain gait variability parameters and quantitative measures including maximum number of continuous steps, maximum walking bout and total continuous walking duration best discriminated non-frail and pre-frail/frail individuals. Step- and stride-time were significantly different between non-frail vs pre-frail/frail $(p<0.001)$. Further, among the frequency domain gait variability parameters, PSD amplitude, PSD slope, and the dominant frequency were significantly different between non-frail and pre-frail/frail groups $(p<0.05)$. However, step- and stridetime variabilities did not show statistical significance between the two groups $(p>0.1)$. Gait asymmetry, representing left and right step coordination, was not statistically significant between the two frailty groups ( $p>0.1)$. Similarly, gait irregularity, measuring predictability of walking cycles, displayed an increasing trend towards pre-frailty/frailty; but the observed between-group differences were not significant $(p>0.1)$. All the continuous walk quantitative measures showed differences between the two groups, and most of these differences were statistically significant between the two groups $(p<0.05)$.

\section{Frailty Prediction using Gait Performance Parameters}

A multinomial logistic regression model was developed using gait performance parameters extracted from DPA along with age and BMI to predict frailty. A step-wise logistic model was developed with frailty 
groups as the dependent variable (non-frail vs. pre-frail/frail), for which, age (years), BMI $\left(\mathrm{kg} / \mathrm{m}^{2}\right)$, stridetime variability (\%), dominant frequency $(\mathrm{Hz})$, and maximum number of continuous steps were selected as independent variables (Table 4). The logistic regression model developed with these features was able to predict pre-frail/frail category with an improved receiver operating characteristics (ROC) area under curve (AUC) of 0.84 compared to age (ROC AUC: 0.71 ) and total number of steps for 48 hours (ROC AUC: 0.77 , Table 5). The ROC curves are shown in Figure 2.

Table 4. Parameter estimates for logistic regression models developed with different parameters

\begin{tabular}{llcccc}
\hline Model Features & Parameter & Parameter Estimate & Std. Error & $\chi 2$ & $p$-value \\
\hline MODEL 1: & Age & -0.0885 & 0.03 & 9.75 & 0.002 \\
Age & & & & & \\
\hline MODEL 2: & Total number of steps & 0.0001 & 0.00 & 12.54 & 0.001 \\
Total number of steps & & & & & \\
\hline MODEL 3: & Age (years) & -0.1191 & 0.04 & 8.27 & 0.004 \\
Gait performance parameters & BMI $\left(\mathrm{kg} / \mathrm{m}^{2}\right)$ & -0.1772 & 0.06 & 8.49 & 0.004 \\
& Stride variability (\%) & -0.2507 & 0.11 & 5.39 & 0.020 \\
& Dominant frequency (Hz) & 6.6265 & 2.14 & 9.62 & 0.002 \\
& Max no. of continuous steps & 0.0001 & 0.00 & 0.33 & 0.565 \\
\hline
\end{tabular}

Table 5. Logistic model performance comparison of different parameters for $80 \%$ specificity

\begin{tabular}{lcccc}
\hline \multicolumn{1}{c}{ Model Features } & Accuracy & Sensitivity & Specificity & AUC \\
\hline Age & $65.1 \%$ & $58.1 \%$ & $80 \%$ & 0.71 \\
Total number of steps & $74.6 \%$ & $46.5 \%$ & $80 \%$ & 0.77 \\
Gait performance parameters & $77.7 \%$ & $76.8 \%$ & $80 \%$ & 0.84 \\
\hline
\end{tabular}

Results of logistic regression models developed with gait performance parameters extracted from $20 \mathrm{~s}$, 30 s, 40 s and 50 s continuous walks, age, and BMI to predict pre-frail/frail category for the different continuous walking lengths are shown in supplementary Table 2 . The corresponding ROC curves in comparison with the $60 \mathrm{~s} \mathrm{ROC}$ curve are presented in supplementary Figure 1. Of note, we observed that among the five different durations (20s, 30s, 40s, 50 s and 60s), gait performance parameters extracted from 60 s continuous walks provided the best frailty assessment results (supplementary Table 2 and supplementary Figure 1).

\section{Discussion}

As hypothesized, several sensor-based gait performance parameters significantly discriminated between non-frail and pre-frail/frail groups, even when adjusted with age. In our previous studies the total number of steps and walking duration could not significantly discriminate pre-frail participants from non-frail when adjusted with age (17). This improvement in frailty assessment occurred due to utilization of the novel concept of continuous walking for obtaining both qualitative and quantitative gait performance parameters. 


\section{Continuous Walks}

The ability to walk longer distances is instrumental for humans to perform various activities of daily living and lead an independent life. Walking requires complex mechanisms within the human sensory motor system to provide the necessary timing, coordination, and balance such that the interplay between the center of mass and the base of support are regulated in a repetitive manner $(54,64)$. Previous studies that explored the gait characteristics of non-disabled adults for two weeks to define walking duration, found that $81 \%$ of all walking bouts lasted about 60 seconds $(35,36)$. Also to assess the effect of continuous walk duration in pre-frailty/frailty assessment, we analyzed gait performance parameters extracted from $20 \mathrm{~s}, 30 \mathrm{~s}, 40 \mathrm{~s}, 50 \mathrm{~s}$, and 60 s continuous walking bouts and observed that 60 s continuous walks provided the best pre-frailty/frailty assessment results compared to other continuous walk durations. Previous studies also showed that accelerometer-derived gait performance measures based on daily activities could improve fall risk evaluation in older adults, when 60-second continuous walking periods were implemented compared to overall number of steps (36). Similarly, in the current study, 60 second continuous walking with no pauses resulted in better discrimination between the frailty groups $(p<0.05)$, when compared with continuous walks with shorter duration and walks that included pauses.

\section{Advantages of Qualitative Gait Parameters}

In addition to previously reported quantitative gait parameters (number of steps, mean walking bout duration, and longest walking bout duration) (17), here we extracted qualitative gait performance parameters (gait variability, asymmetry, and irregularity). Specifically, gait variability represented a promising measure for differentiating gait deficits among the three frailty categories. Gait variability, defined as the stride-to-stride fluctuation in walking cycles, has been previously associated with high risk of fall and cognitive impairments in elders $(6,39-41,65)$. Gait variability reflects inconsistency in physiological systems that regulate walking, including neuromuscular, reflexive postural control, and cardiovascular systems. We used two methods to assess gait variability: step/stride time variability using time-domain and power spectral density (PSD) using frequency-domain analyses. We observed that the frequency-domain parameters were significantly different between frailty groups, while time-domain parameters were not. Owing to the low sampling frequency in this study, some of the information content may be lost due to filtering for peak detection (66). Hence, the PSD analysis performed on the entire raw acceleration signal may provide a more efficient tool for assessing gait variability for low sampling frequency motion sensor data.

Additionally, gait asymmetry and irregularity were also investigated here, as parameters that represent gait deficits independent of gait variability. Gait asymmetry, representing left-right step coordination, has been used as a metric to observe walking patterns in older individuals. Cognitive aging and sarcopenia render gait to be less automatic and left-right symmetry co-ordination is expected to require additional effort, especially in frail individuals (37). Although gait asymmetry showed a decreasing trend from nonfrail to pre-frail and frail samples, differences were not significant (Table 3). Since loss in stepcoordination may also happen due to hip, knee, or ankle impairments (67), these confounding variables 
can mask the effect of frailty. Further, gait irregularity, representing the predictability of walking cycles, can be influenced by both neurological and neuromuscular diseases (18,50-52). Previous studies have used sample entropy to obtain gait predictability or repeatability to investigate differences in the relationship between executive function, and gait variability and stability during single and dual-task walking in persons with and without dementia (51). We computed gait irregularity using the sample entropy method and observed an increase in irregularity in pre-frail/frail population but there was no statistical significance seen between the groups (Table 3).

\section{Limitations}

There are limitations to consider in the interpretation of these findings. The first limitation is the lack of longitudinal validation of the DPA index for direct prediction of frailty-related health complications. Accordingly, due to a cross-sectional design of the current study, no conclusion can be made regarding the accuracy of the proposed index compared to the Fried test, or other types of frailty measures. Second limitation is adherence to the wearable sensor equipment. Though the participants did not express any obvious discomfort while wearing the sensors, it is possible that a few of the participants forgot to wear them immediately after a shower. Third, our sample was predominantly women. Although we did not observe a gender specific difference in gait performance, the model developed here may have limited generalizability to a population with a more balanced gender composition. Fourth, we consider a continuous walk as one with $20 / 30 / 40 / 50 / 60$ seconds or longer duration with no pauses. However, it is possible that this walk was purposeful with the individual walking to a certain destination or that this walk was a random stroll with no specific purpose. These possibilities could potentially bias the quality of walking, but in our data we did not have the required information to categorize continuous walks as purposeful or random. Finally, not all participants, especially pre-frail and frail ones, had continuous walks during the 48 hours of data collection, causing a reduced sample size for the pre-frail/frail group. To overcome this limitation, we combined pre-frail and frail participants and all the participants who did not exhibit continuous walking were automatically categorized as pre-frail/frail while developing the logistic model. Since detecting the onset of frailty at the pre-frail stage is most crucial in recovering health status (68), our findings suggested a promising method for pre-frailty identification using DPA data.

\section{Summary And Conclusion}

Using gait performance parameters extracted from 60 s continuous walks within 48 hours of daily monitoring, pre-frailty/frailty was identified with a sensitivity of $76.8 \%$ and specificity of $80 \%$ among elders. Findings suggest that these DPA-based 60 s continuous walking parameters, including gait variability and the amount of continuous walking, may noticeably improve gait deficit assessment compared to previous in-clinic gait assessment methods. The proposed gait performance characterization based on sensor-based daily physical activity provides potential for being integrated into clinical care for in-home screening of frailty (much as a holter monitor is used) to provide information pertaining to an individual's condition before hospital admission, or when frailty is suspected. This method is advantageous over its in-clinic counterpart as it is objective rather than subjective self-reported 
measures of physical activity, and is measured in real-world walking activities rather than an artificial clinical setting. Although Fried's frailty criteria (2) provides an accurate frailty measure and we observed strong association with this gold standard, the DPA frailty index should be further validated within longitudinal settings for predicting adverse health outcomes among older adults. Nevertheless, our proposed technique eliminates the bias pertaining to self-reported measures, does not require the subject to commute to the clinic, and provides a continuous in-home assessment.

Healthcare research in wearable devices has been constantly growing in various areas like remote patient monitoring and healthcare (69-73), wearable sensor-based systems for health monitoring (74-77), and ambient-assisted living tools for older adults (78). For the older adults requiring continuous health monitoring, sensor-based wearables and remote monitoring will help eliminate the hassle of periodic commute to diagnostic centers, reduce the amount of recurring admissions to the hospital, and facilitate more efficient clinical visits with objective results (79).

\section{Abbreviations}

ADL: Barthel activity of daily living; AIC: Akaike information criteria; ANOVA: Analysis of variance; AUC: Area under the curve; BMI: Body mass index; CES-D: Center for Epidemiologic Studies Depression Scale; CGA - Comprehensive Geriatric Assessment; DPA: Daily physical activity; MMSE: Mini-mental state examination; PSD: Power spectral density; ROC: Receiver operating characteristic; SampEn: Sample entropy; SD: Standard deviation; VIF: Variance inflation factor; WHO: World health organization.

\section{Declarations}

\section{Ethics approval and consent to participate}

The study was approved by the University of Arizona and Banner Sun Health Research Institute's Institutional Review Boards. Before participation, written informed consent according to the principles expressed in the Declaration of Helsinki was obtained from all subjects.

\section{Consent for publication}

Not applicable.

\section{Availability of data and materials}

The datasets used and/or analyzed during the current study available from the corresponding author on reasonable request.

\section{Competing interests}

The authors declare that they have no competing interests. 


\section{Funding}

This study was supported in part by award No. 2R42AG032748 from the National Institute on Aging. Sponsors had no role in the design of the study and collection, analysis, and interpretation of data and in writing the manuscript.

\section{Authors' contributions}

DPK: data analysis, interpretation of findings, statistical analysis, manuscript writing and critical review of manuscript. NT: concept, study design, subject recruitment, study management, data analysis and interpretation, statistical analysis, manuscript writing and editing, critical review of manuscript and approval of final manuscript. JM: subject recruitment, study management, study design, interpretation of findings, critical review of manuscript and approval of final manuscript. HE: interpretation of findings, critical review of manuscript, comments and approval of final manuscript. CM: data analysis and interpretation. KL: concept, study design, data analysis and interpretation, statistical analysis, manuscript writing and editing, critical review of manuscript and approval of final manuscript.

\section{Acknowledgements}

We thank the students of University of Arizona and Arizona Frailty Cohort coordination staff for volunteer recruitment and data collection.

\section{References}

1. Mohler MJ, Fain MJ, Wertheimer AM, Najafı B, Nikolich-Žugich J. The Frailty Syndrome: Clinical measurements and basic underpinnings in humans and animals. Exp Gerontol. 2014 Jun;54:6-13.

2. Fried LP, Tangen CM, Walston J, Newman AB, Hirsch C, Gottdiener J, et al. Frailty in Older Adults: Evidence for a Phenotype. Journals Gerontol Ser A Biol Sci Med Sci. 2001 Mar 1;56(3):M146-57.

3. Evans WJ, Paolisso G, Abbatecola AM, Corsonello A, Bustacchini S, Strollo F, et al. Frailty and muscle metabolism dysregulation in the elderly. Biogerontology. 2010 Oct 4;11(5):527-36.

4. van Kan GA, Rolland $Y$, Houles M, Gillette-Guyonnet $S$, Soto $M$, Vellas $B$. The assessment of frailty in older adults. Vol. 26, Clinics in Geriatric Medicine. 2010. p. 275-86.

5. Abellan Van Kan G, Rolland Y, Bergman H, Morley JE, Kritchevsky SB, Vellas B. The I.A.N.A. task force on frailty assessment of older people in clinical practice. J Nutr Heal Aging. 2008 Jan;12(1):29-37.

6. Hausdorff JM, Edelberg HK, Mitchell SL, Goldberger AL, Wei JY. Increased gait unsteadiness in community-dwelling elderly failers. Arch Phys Med Rehabil. 1997 Mar;78(3):278-83.

7. Lipsitz LA. Dynamics of stability: the physiologic basis of functional health and frailty. J Gerontol A Biol Sci Med Sci. 2002 Mar;57(3):B115-25.

8. Grabiner PC, Biswas ST, Grabiner MD. Age-related changes in spatial and temporal gait variables. Arch Phys Med Rehabil. 2001 Jan;82(1):31-5. 
9. Guralnik JM, Ferrucci L, Simonsick EM, Salive ME, Wallace RB. Lower-Extremity Function in Persons over the Age of 70 Years as a Predictor of Subsequent Disability. N Engl J Med. 1995 Mar 2;332(9):556-62.

10. Visser M, Deeg DJH, Lips P, Longitudinal Aging Study Amsterdam. Low Vitamin D and High Parathyroid Hormone Levels as Determinants of Loss of Muscle Strength and Muscle Mass (Sarcopenia): The Longitudinal Aging Study Amsterdam. J Clin Endocrinol Metab. 2003 Dec;88(12):5766-72.

11. Martinikorena I, Martínez-Ramírez A, Gómez M, Lecumberri P, Casas-Herrero A, Cadore EL, et al. Gait Variability Related to Muscle Quality and Muscle Power Output in Frail Nonagenarian Older Adults. J Am Med Dir Assoc. 2016 Feb 1;17(2):162-7.

12. Kosse NM, Vuillerme N, Hortobágyi T, Lamoth CJ. Multiple gait parameters derived from iPod accelerometry predict age-related gait changes. Gait Posture. 2016 May;46:112-7.

13. Rockwood K, Stadnyk K, MacKnight C, McDowell I, Hébert R, Hogan DB. A brief clinical instrument to classify frailty in elderly people. Lancet. 1999 Jan 16;353(9148):205-6.

14. Mitnitski AB, Graham JE, Mogilner AJ, Rockwood K. Frailty, fitness and late-life mortality in relation to chronological and biological age. BMC Geriatr. 2002 Feb 27;2:1-8.

15. Jones DM, Song $X$, Rockwood K. Operationalizing a frailty index from a standardized comprehensive geriatric assessment. J Am Geriatr Soc. 2004 Nov;52(11):1929-33.

16. Paw MJMCA, De Groot LCPGM, Van Gend S V., Schoterman MHC, Schouten EG, Schroll M, et al. Inactivity and weight loss: Effective criteria to identify frailty. J Nutr Heal Aging. 2003;7(1):55-60.

17. Schwenk M, Mohler J, Wendel C, D"Huyvetter K, Fain M, Taylor-Piliae R, et al. Wearable Sensor-Based In-Home Assessment of Gait, Balance, and Physical Activity for Discrimination of Frailty Status: Baseline Results of the Arizona Frailty Cohort Study. Gerontology. 2015;61(3):258-67.

18. Lamoth CJ, van Deudekom FJ, van Campen JP, Appels BA, de Vries OJ, Pijnappels M. Gait stability and variability measures show effects of impaired cognition and dual tasking in frail people. J Neuroeng Rehabil. 2011 Dec;8(1):2.

19. Menz HB, Lord SR, Fitzpatrick RC. Acceleration patterns of the head and pelvis when walking on level and irregular surfaces. Gait Posture. 2003 Aug;18(1):35-46.

20. Lindemann U, Najafi B, Zijlstra W, Hauer K, Muche R, Becker C, et al. Distance to achieve steady state walking speed in frail elderly persons. Gait Posture. 2008 Jan;27(1):91-6.

21. Zhong R, Rau P-LP, Yan X. Application of smart bracelet to monitor frailty-related gait parameters of older Chinese adults: A preliminary study. Geriatr Gerontol Int. 2018 Sep;18(9):1366-71.

22. Martínez-Ramírez A, Martinikorena I, Gómez M, Lecumberri P, Millor N, Rodríguez-Mañas L, et al. Frailty assessment based on trunk kinematic parameters during walking. J Neuroeng Rehabil. 2015 May 24;12:48.

23. Folstein MF, Folstein SE, McHugh PR. \&quot;Mini-mental state\&quot;. A practical method for grading the cognitive state of patients for the clinician. J Psychiatr Res. 1975 Nov;12(3):189-98. 
24. World Medical Association. World Medical Association Declaration of Helsinki. JAMA. 2013 Nov 27;310(20):2191.

25. Fieo RA, Mortensen EL, Rantanen T, Avlund K, Fieo R. Improving a Measure of Mobility-Related Fatigue (The Mobility-Tiredness Scale) by Establishing Item Intensity. J Am Geriatr Soc. 2013;61(3):429-33.

26. Orme JG, Reis J, Herz EJ. Factorial and discriminant validity of the Center for Epidemiological Studies Depression (CES-D) scale. J Clin Psychol. 1986 Jan;42(1):28-33.

27. Yardley L, Beyer N, Hauer K, Kempen G, Piot-Ziegler C, Todd C. Development and initial validation of the Falls Efficacy Scale-International (FES-I). Age Ageing. 2005 Nov 1;34(6):614-9.

28. MAHONEY FI, BARTHEL DW. FUNCTIONAL EVALUATION: THE BARTHEL INDEX. Md State Med J. 1965 Feb;14:61-5.

29. Najafı B, Aminian K, Paraschiv-lonescu A, Loew F, Bula CJ, Robert P. Ambulatory system for human motion analysis using a kinematic sensor: monitoring of daily physical activity in the elderly. IEEE Trans Biomed Eng. 2003 Jun;50(6):711-23.

30. Najafı B, Aminian K, Loew F, Blanc Y, Robert PA. Measurement of stand-sit and sit-stand transitions using a miniature gyroscope and its application in fall risk evaluation in the elderly. IEEE Trans Biomed Eng. 2002 Aug;49(8):843-51.

31. Najafi B, Armstrong DG, Mohler J. Novel Wearable Technology for Assessing Spontaneous Daily Physical Activity and Risk of Falling in Older Adults with Diabetes. J Diabetes Sci Technol. 2013 Sep 1;7(5):1147-60.

32. Ismail AR, Asfour SS. Discrete wavelet transform: a tool in smoothing kinematic data. J Biomech. 1999 Mar 1;32(3):317-21.

33. Wachowiak MP, Rash GS, Quesada PM, Desoky AH. Wavelet-based noise removal for biomechanical signals: A comparative study. IEEE Trans Biomed Eng. 2000;47(3):360-8.

34. Sekine M, Tamura T, Ogawa M, Togawa T, Fukui Y. Classification of acceleration waveform in a continuous walking record. In Institute of Electrical and Electronics Engineers (IEEE); 2002. p. 15236.

35. Orendurff MS. How humans walk: Bout duration, steps per bout, and rest duration. J Rehabil Res Dev. 2008 Dec 1;45(7):1077-90.

36. Weiss A, Brozgol M, Dorfman M, Herman T, Shema S, Giladi N, et al. Does the Evaluation of Gait Quality During Daily Life Provide Insight Into Fall Risk? A Novel Approach Using 3-Day Accelerometer Recordings. Neurorehabil Neural Repair. 2013 Oct 17;27(8):742-52.

37. Yogev G, Plotnik M, Peretz C, Giladi N, Hausdorff JM. Gait asymmetry in patients with Parkinson's disease and elderly fallers: when does the bilateral coordination of gait require attention? Exp Brain Res. 2007 Feb 15;177(3):336-46.

38. John D, Miller R, Kozey-Keadle S, Caldwell G, Freedson P. Biomechanical Examination of the 'Plateau Phenomenon' in ActiGraph Vertical Activity Counts. Physiol Meas. 2012 Feb;33(2):219. 
39. Hausdorff JM, Rios DA, Edelberg HK. Gait variability and fall risk in community-living older adults: A 1-year prospective study. Arch Phys Med Rehabil. 2001 Aug;82(8):1050-6.

40. Sheridan PL, Solomont J, Kowall N, Hausdorff JM. Influence of Executive Function on Locomotor Function: Divided Attention Increases Gait Variability in Alzheimer's Disease. J Am Geriatr Soc. 2003 Nov;51(11):1633-7.

41. Verghese J, Holtzer R, Lipton RB, Wang C. Mobility Stress Test Approach to Predicting Frailty, Disability, and Mortality in High-Functioning Older Adults. J Am Geriatr Soc. 2012 Oct;60(10):19015.

42. Hausdorff JM. Gait dynamics, fractals and falls: finding meaning in the stride-to-stride fluctuations of human walking. Hum Mov Sci. 2007 Aug;26(4):555-89.

43. Toosizadeh N, Lei H, Schwenk M, Sherman SJ, Sternberg E, Mohler J, et al. Does Integrative Medicine Enhance Balance in Aging Adults? Proof of Concept for the Benefit of Electroacupuncture Therapy in Parkinson's Disease. Gerontology. 2015;61(1):3-14.

44. Lei H, Toosizadeh N, Schwenk M, Sherman S, Karp S, Sternberg E, et al. A Pilot Clinical Trial to Objectively Assess the Efficacy of Electroacupuncture on Gait in Patients with Parkinson's Disease Using Body Worn Sensors. PLoS One. 2016;11(5).

45. Weiss A, Sharifi S, Plotnik M, van Vugt JPP, Giladi N, Hausdorff JM. Toward Automated, At-Home Assessment of Mobility Among Patients With Parkinson Disease, Using a Body-Worn Accelerometer. Neurorehabil Neural Repair. 2011 Nov 11;25(9):810-8.

46. Petre Stoica \& Randolph L. Moses. Spectral Analysis of Signals | Pearson. Pearson; 2005.

47. Toosizadeh N, Mohler J, Najafi B. Assessing Upper Extremity Motion: An Innovative Method to Identify Frailty. J Am Geriatr Soc. 2015 Jun;63(6):1181-6.

48. Palshikar GK. Simple Algorithms for Peak Detection in Time-Series Simple Algorithms for Peak Detection in Time-Series. Design. 2002;(July):1-13.

49. Lamoth CJ, van Deudekom FJ, van Campen JP, Appels BA, de Vries OJ, Pijnappels M. Gait stability and variability measures show effects of impaired cognition and dual tasking in frail people. $\mathrm{J}$ Neuroeng Rehabil. 2011 Dec 17;8(1):2.

50. Liao F, Wang J, He P. Multi-resolution entropy analysis of gait symmetry in neurological degenerative diseases and amyotrophic lateral sclerosis. Med Eng Phys. 2008 Apr;30(3):299-310.

51. IJmker T, Lamoth CJC. Gait and cognition: The relationship between gait stability and variability with executive function in persons with and without dementia. Gait Posture. 2012 Jan;35(1):126-30.

52. Riva F, Toebes MJP, Pijnappels M, Stagni R, van Dieën JH. Estimating fall risk with inertial sensors using gait stability measures that do not require step detection. Gait Posture. 2013 Jun;38(2):170-4.

53. Yentes JM, Hunt N, Schmid KK, Kaipust JP, McGrath D, Stergiou N. The Appropriate Use of Approximate Entropy and Sample Entropy with Short Data Sets. Ann Biomed Eng. 2013 Feb 12;41(2):349-65. 
54. Orter S, Ravi DK, Singh NB, Vogl F, Taylor WR, König Ignasiak N. A method to concatenate multiple short time series for evaluating dynamic behaviour during walking. PLoS One. 2019;14(6):e0218594.

55. McCamley JD, Denton W, Arnold A, Raffalt PC, Yentes JM. On the calculation of sample entropy using continuous and discrete human gait data. Entropy (Basel). 2018 Oct;20(10).

56. Raffalt PC, McCamley J, Denton W, Yentes JM. Sampling frequency influences sample entropy of kinematics during walking. Med Biol Eng Comput. 2019 Apr 3;57(4):759-64.

57. Shi L, Duan F, Yang Y, Sun Z. The Effect of Treadmill Walking on Gait and Upper Trunk through Linear and Nonlinear Analysis Methods. Sensors (Basel). 2019 May 13;19(9).

58. Albers DJ, Hripcsak G. Estimation of time-delayed mutual information and bias for irregularly and sparsely sampled time-series. Chaos Solitons Fractals. 2012 Jun 1;45(6):853-60.

59. Lipsitz LA, Goldberger AL. Loss of "complexity" and aging. Potential applications of fractals and chaos theory to senescence. JAMA. 1992 Apr 1;267(13):1806-9.

60. Moe-Nilssen R, Helbostad JL. Estimation of gait cycle characteristics by trunk accelerometry. J Biomech. 2004 Jan 1;37(1):121-6.

61. Richman JS, Lake DE, Moorman JR. Sample Entropy. In: Methods in enzymology. 2004. p. 172-84.

62. Richman JS, Moorman JR. Physiological time-series analysis using approximate entropy and sample entropy. Am J Physiol Circ Physiol. 2000 Jun;278(6):H2039-49.

63. O'brien RM. A Caution Regarding Rules of Thumb for Variance Inflation Factors. 2007;

64. Hof AL. The 'extrapolated center of mass' concept suggests a simple control of balance in walking. Hum Mov Sci. 2008 Feb;27(1):112-25.

65. Toosizadeh N, Mohler J, Lei H, Parvaneh S, Sherman S, Najafi B. Motor Performance Assessment in Parkinson's Disease: Association between Objective In-Clinic, Objective In-Home, and Subjective/Semi-Objective Measures. Maetzler W, editor. PLoS One. 2015 Apr 24;10(4):e0124763.

66. Proakis, John G; Manolakis DG. Digital signal processing: Principles, algorithms, and applications. 1992.

67. Casartelli NC, Item-Glatthorn JF, Bizzini M, Leunig M, Maffiuletti NA. Differences in gait characteristics between total hip, knee, and ankle arthroplasty patients: a six-month postoperative comparison. BMC Musculoskelet Disord. 2013 Jun 3;14:176.

68. Gill TM, Gahbauer EA, Allore HG, Han L. Transitions Between Frailty States Among Community-Living Older Persons. Arch Intern Med. 2006 Feb 27;166(4):418.

69. Suh M, Chen C-A, Woodbridge J, Tu MK, Kim JI, Nahapetian A, et al. A Remote Patient Monitoring System for Congestive Heart Failure. J Med Syst. 2011 Oct 25;35(5):1165-79.

70. Youm S, Lee G, Park S, Zhu W. Development of remote healthcare system for measuring and promoting healthy lifestyle. Expert Syst Appl. 2011 Mar 1;38(3):2828-34.

71. Malhi K, Mukhopadhyay SC, Schnepper J, Haefke M, Ewald H. A Zigbee-Based Wearable Physiological Parameters Monitoring System. IEEE Sens J. 2012 Mar;12(3):423-30. 
72. Yamada I, Lopez G. Wearable sensing systems for healthcare monitoring. In: 2012 Symposium on VLSI Technology (VLSIT). IEEE; 2012. p. 5-10.

73. Custodio V, Herrera F, López G, Moreno J, Custodio V, Herrera FJ, et al. A Review on Architectures and Communications Technologies for Wearable Health-Monitoring Systems. Sensors. 2012 Oct 16;12(10):13907-46.

74. Pantelopoulos A, Bourbakis NG. A Survey on Wearable Sensor-Based Systems for Health Monitoring and Prognosis. Appl Rev. 2010;40(1).

75. Alemdar H, Ersoy C. Wireless sensor networks for healthcare: A survey. Comput Networks. 2010 Oct 28;54(15):2688-710.

76. Baig MM, Gholamhosseini H. Smart Health Monitoring Systems: An Overview of Design and Modeling. J Med Syst. 2013 Apr 15;37(2):9898.

77. Chuan Yen T, Mohler J, Dohm M, Laksari K, Najafı B, Toosizadeh N. The Effect of Pain Relief on Daily Physical Activity: In-Home Objective Physical Activity Assessment in Chronic Low Back Pain Patients after Paravertebral Spinal Block. Sensors. 2018 Sep 12;18(9):3048.

78. Rashidi P, Mihailidis A. A Survey on Ambient-Assisted Living Tools for Older Adults. IEEE J Biomed Heal Informatics. 2013 May;17(3):579-90.

79. Scanaill CN, Carew S, Barralon P, Noury N, Lyons D, Lyons GM. A Review of Approaches to Mobility Telemonitoring of the Elderly in Their Living Environment. Ann Biomed Eng. 2006 Apr 21;34(4):54763.

\section{Figures}
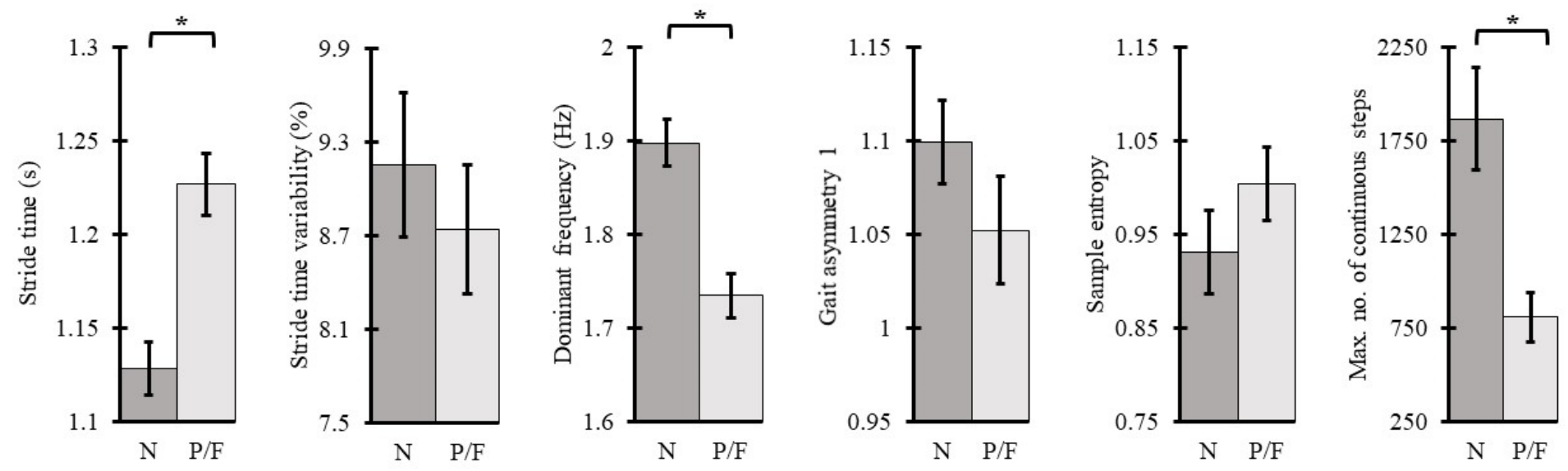

\section{Figure 1}

Comparison of continuous gait performance parameters between non-frail $(\mathrm{N})$ and pre-frail($(\mathrm{P}) / \mathrm{frail}(\mathrm{F})$ groups $\left({ }^{\star} p<0.05\right)$ 


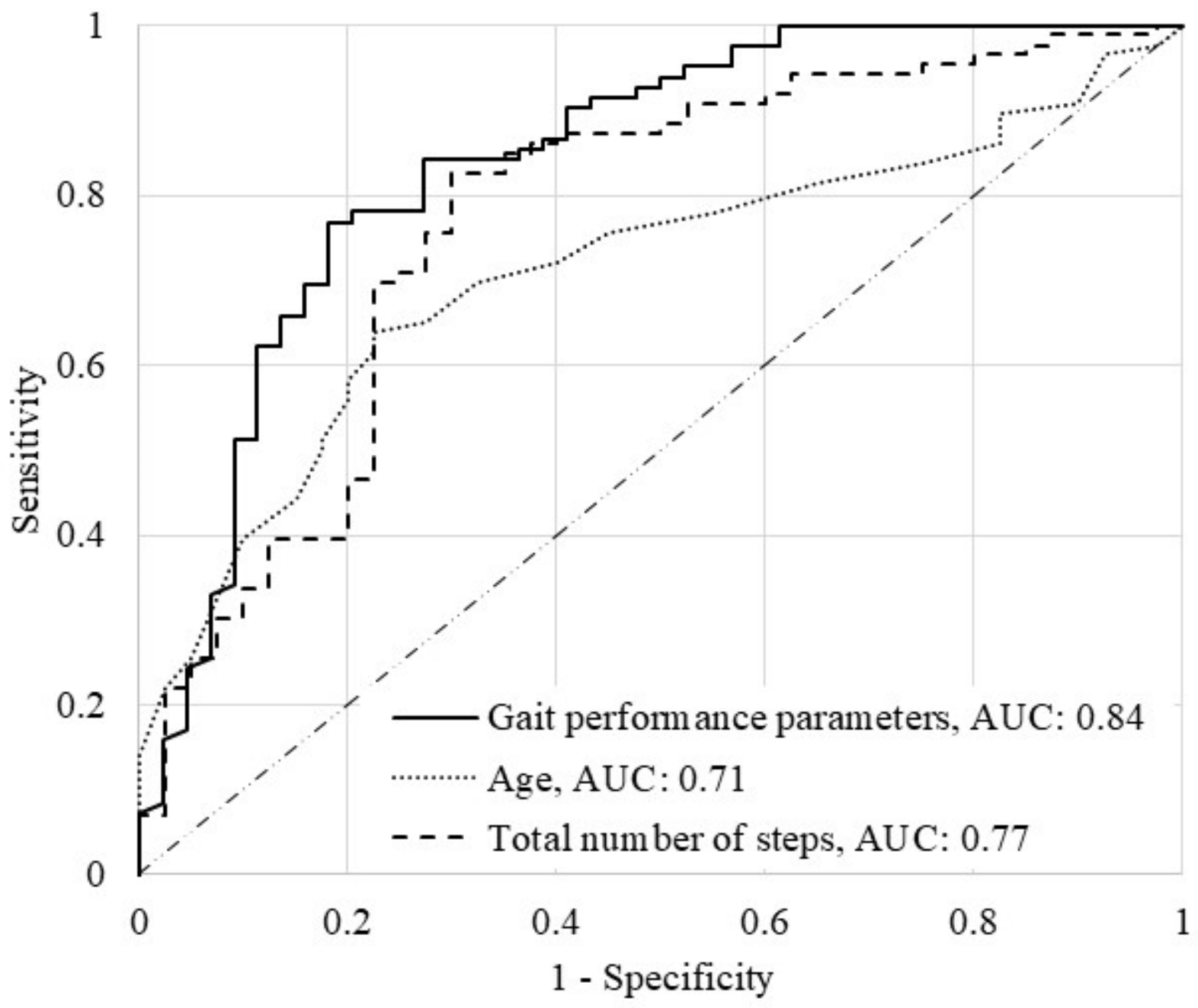

Figure 2

Logistic regression model ROC curves for age, total number of steps, and gait performance parameters

\section{Supplementary Files}

This is a list of supplementary files associated with this preprint. Click to download.

- SupplememtarymaterialDPApaperBMCgeriatrics.pdf

- SupplementaryFigure1.tif

- Equations.pdf 